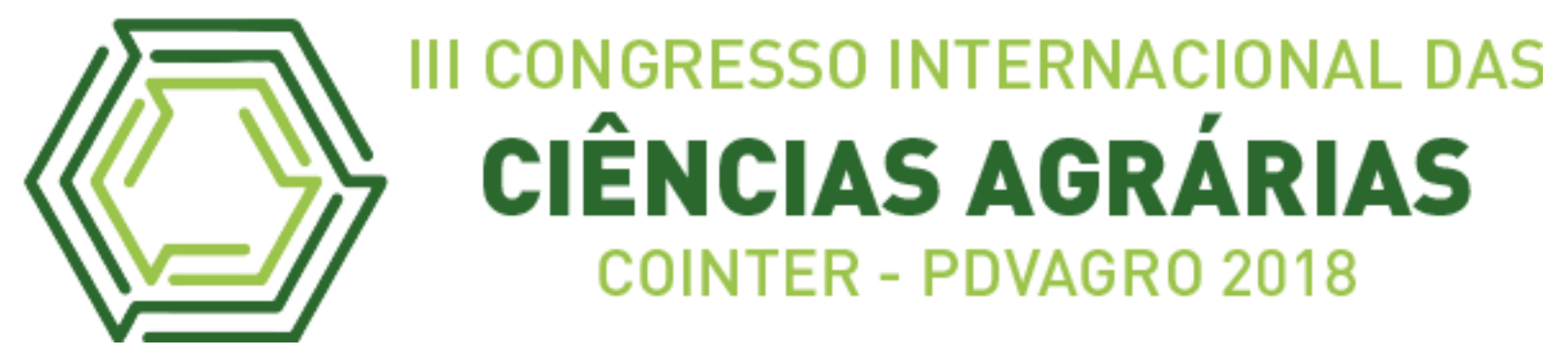

\title{
TÉCNICAS MULTIVARIADAS NA AVALIAÇÃO DE ATRIBUTOS DO SOLO COMO INDICADOR DE SUSTENTABILIDADE
}

\section{MULTIVARIATED TECHNIQUES EVALUATION OF SOIL ATTRIBUTES FOR A SUSTAINABILITY INDICATOR}

\author{
Apresentação: Pôster \\ Nayane Jaqueline Costa Maia ${ }^{1}$; Whesley Thiago dos Santos Lobato ${ }^{2}$; Gabriela Mourão \\ de Almeida ${ }^{3}$
}

DOI: https://doi.org/10.31692/2526-7701.IIICOINTERPDVAGRO.2018.00671

\section{Introdução}

A conservação do solo constitui-se de um dos grandes desafios do Século XXI, pois a degradação deste recurso pode levar a ocorrência de diversos problemas ambientais. Por esse motivo, para conservar o solo é importante conhecer e entender o conhecimento de educação ambiental. E esse processo de formação de conhecimento ambiental precisa ser dinâmico, permanente e participativo (PEREIRA; CURI, 2012; MUGGLER et al., 2006).

$\mathrm{O}$ estudo do comportamento dos atributos do solo ao longo do tempo permite quantificar a magnitude e a duração das alterações provocadas por diferentes sistemas de manejo, conforme destacam Reichert et al., (2009), segundo os quais, por serem parâmetros sensíveis, essas propriedades são importantes para estabelecer se houve degradação ou melhoria da qualidade do solo, em referência a um sistema de manejo determinado.

O objetivo deste trabalho foi avaliar as alterações nos atributos químicos, físicos e microbiológicos de um Latossolo Amarelo distrófico e um Neossolo Flúvico distrófico submetido à sistemas de manejos, com o uso de técnicas de estatística multivariada.

\section{Fundamentação teórica}

\footnotetext{
1 Mestranda em Agronomia, Universidade Estadual Paulista - Campus de Jaboticabal, nayane.maia1@gmail.com

2 Discente de Agronomia, Universidade Federal Rural da Amazônia - Campus Capitão Poço, whesleylobatospfc@gmail.com

${ }^{3}$ Mestranda em Agronomia, Universidade Estadual Paulista - Campus de Jaboticabal, gabrielamouraodealmeida@gmail.com
} 
As análises estatísticas convencionais não são, muitas vezes, suficientes para descrever as interações neste sistema (SILVA et al., 2010a). Uma das soluções propostas é a utilização da estatística multivariada por possuir maior capacidade de descrever as relações de intra e interdependência nos sistemas agrícolas (MARQUES JÚNIOR, 2009).

Apesar da grande importância dos métodos estatísticos multivariados para interpretações das variações dos atributos do solo, poucos são os trabalhos que fazem uso desta ferramenta, pois a maioria utiliza métodos estatísticos univariados (SILVA et al., 2010b). No entanto, alguns estudos têm aplicado técnicas multivariadas para avaliação das variáveis do solo e encontrado resultados satisfatórios (CAMPOS et al., 2012; OLIVEIRA, 2013).

\section{Metodologia}

O trabalho foi desenvolvido no município de Castanhal, nordeste paraense do Brasil, na comunidade de Macapazinho. Durante os anos de 2015 e 2016 foram realizadas coletas de solos em duas grandes áreas: A primeira área é formada por um Latossolo Amarelo distrófico (LAD), tem uma classe textural arenosa, formada por solos de sequeiro. A segunda área é formada por um Neossolo Flúvico distrófico (NFD) formada em sua maioria por solos alagados, denominada de várzea, e sua classe textural é areno-argilosa.

Nos dois solos estudados foram realizadas as amostragens de solos nas profundidades de 0,0-0,2 m, visando compor as amostras deformadas e indeformadas. Para as análises dos atributos microbiológicos, a amostragem do solo foi realizada na profundidade de $0,0-0,10 \mathrm{~m}$.

As análises de solos foram realizadas segundo o manual de análises química e físicas (SILVA, 2009), das quais foram realizadas as análises de: Matéria Orgânica, Nitrogênio Total, Cálcio e Magnésio, Alumínio, Fósforo, acidez do solo (pH) (Tabela 1). As análises físicas que foram realizadas, são: densidade do solo, densidade das partículas e porosidade total. A partir desses resultados dos atributos físicos do solo foi possível determina a umidade gravimétrica do solo (Tabela 2).

O carbono da biomassa microbiana do solo (C-BMS) foi analisado pelo método de fumigação-extração-titulação modificado por Silva et al. (2007a). O nitrogênio da biomassa microbiana do solo (N-BMS) foi analisado pelo método de fumigação-extração-destilaçãotitulação modificado por Silva et al. (2007b). Os resultados do Carbono-BMS ficaram na média de $93 \mathrm{~g} \mathrm{~kg}^{-1}$ de solo para o NFD, e $65 \mathrm{~g} \mathrm{~kg}^{-1}$ de solo para o LAD. Os resultados obtidos 
de Nitrogênio-BMS ficaram na média de $5 \mathrm{~g} \mathrm{~kg}^{-1}$ de solo para o NFD, e 4,5 $\mathrm{g} \mathrm{kg}^{-1}$ de solo para o LAD.

Tabela 1. Atributos químicos do solo nas áreas estudadas, região de Castanhal, PA.

\begin{tabular}{|c|c|c|c|c|c|c|c|c|}
\hline \multicolumn{9}{|c|}{ Atributos Químicos } \\
\hline \multirow{2}{*}{ Áreas } & MO & pH & $\mathbf{P}$ & $\mathbf{N}$ & $\mathbf{C a}$ & $\mathbf{M g}$ & Al & $\mathbf{H}+\mathbf{A l}$ \\
\hline & $g \mathbf{d m}^{-3}$ & $\mathrm{H}_{2} \mathrm{O}$ & $\mathbf{m g ~ d m ^ { - 3 }}$ & $\%$ & \multicolumn{4}{|c|}{$\operatorname{mmol}_{\mathrm{c}} \mathrm{dm}^{-3}$} \\
\hline & \multicolumn{8}{|c|}{ camada de $0,0-0,2 \mathrm{~m}$} \\
\hline $\mathrm{NFD}^{(1)}$ & 1,12 & 5,1 & 4,06 & 0,18 & 0,5 & 0,5 & 0,7 & 3,7 \\
\hline $\operatorname{LAD}^{(2)}$ & 1,11 & 4,7 & 0,94 & 0,16 & 0,6 & 0,4 & 1 & 5,2 \\
\hline
\end{tabular}

Médias de quatro repetições; ${ }^{(1)}$ Neossolo Flúvico Distrófico; ${ }^{(2)}$ Latossolo Amarelo Distrófico. $\mathrm{pH}, \mathrm{MO}=$ matéria orgânica; $\mathrm{P}=$ fósforo; $\mathrm{N}=$ nitrogênio; $\mathrm{Ca}=$ cálcio, $\mathrm{Mg}=$ magnésio, $\mathrm{H}+\mathrm{Al}=$ acidez potencial, $\mathrm{Al}=$ =alumínio.

Tabela 2. Atributos físicos do solo nas áreas estudadas, região de Castanhal, PA.

\begin{tabular}{|c|c|c|c|c|}
\hline & \multicolumn{4}{|c|}{ Atributos Físicos } \\
\hline \multirow{2}{*}{ Áreas } & DS & DP & PT & UG \\
\hline & \multicolumn{2}{|c|}{$\mathrm{kg} \mathrm{cm}^{-3}$} & \multicolumn{2}{|c|}{$\%$} \\
\hline & \multicolumn{4}{|c|}{ camada de $0,0-0,2 \mathrm{~m}$} \\
\hline $\mathrm{NFD}^{(1)}$ & 1,45 & 2,63 & 45 & 0,28 \\
\hline $\mathrm{LAD}^{(2)}$ & 1,32 & 2,62 & 49 & 0,18 \\
\hline
\end{tabular}

Médias de quatro repetições; ${ }^{(1)}$ Neossolo Flúvico Distrófico; ${ }^{(2)}$ Latossolo Amarelo Distrófico. DS=densidade do solo, $\mathrm{DP}=$ densidade das partículas, $\mathrm{PT}=$ porosidade total, $\mathrm{UG}=$ umidade gravimétrica.

Os dados foram submetidos à estatística descritiva para a obtenção das médias de cada atributo por uso do solo e à análise estatística multivariada. A fim de identificar a similaridade das áreas estudadas utilizou-se, como ferramenta, a análise de agrupamento (análise de cluster) e o algoritmo de Ward. Os grupos foram definidos pelo traçado de uma linha paralela ao eixo horizontal, em que se encontram as maiores distâncias cujos grupos foram formados.

O dendrograma obtido pela análise de agrupamento apresenta, no eixo vertical, o nível de similaridade e, no eixo horizontal, as áreas, formando as classes homogêneas. À medida que o nível de fusão aumenta, o nível de similaridade decresce. Então, traçar uma linha horizontal no dendrograma significa traçar a Linha Fenon, como é chamada, o que delimitará o número de grupos a se formar. A análise estatística multivariada foi processada no software Minitab®, versão 2018.

\section{Resultados e Discussões}

Com a análise de agrupamento hierárquico (Figura 1), obtido da matriz de dados padronizados, objetivou-se avaliar a semelhança das áreas de estudo. Cada vez que se obtém variação expressiva nos valores de distância euclidiana entre os acessos, para o conjunto de 
variáveis consideradas, é possível fazer uma divisão de grupos. A referida divisão mostrou uma ordenação dos acessos, segundo a qualidade do solo.

Os atributos foram agrupados com base no seu grau de semelhança, com o objetivo de classificá-las em grupos mais ou menos homogêneos. Observa-se que todos os atributos foram agrupados na área 1 (NFD), porque apresentaram maior semelhança por possuírem a menor distância euclidiana.

Após a divisão dos dois tipos de solos, analisando a figura 1, fica claro que o solo de várzea (NFD) englobou os dados formados pelo solo de terra firme (LAD). É obvio que a sustentabilidade de área de várzea tem diferença em relação a área de terra firme nos atributos analisados, isso ocorre por diversos fatores, como disponibilidade de água, e etc.

Entretanto, pode-se observar que a concentração de atributos físicos e químicos do solo ficaram concentrados na área 2 (LAD), isso mostra que um bom manejo do solo é suficiente para melhorar a estrutura e fertilidade do solo, quando comparado com uma área de referência da região amazônica (agroecossistema de várzea), percebe-se a obtenção de uma maior similaridade entre esses dois grupos.

O LAD é formado por um agroecossistema composto por plantio da cultura de Açaí de sequeiro (Euterpe oleraceae), Mandioca (Manihot esculenta), Mangostão (Garcinia mangostana L.), Banana (Musa ssp.), Cupuaçu (Theobrama glandiflorum), Castanha-do-Pará (Bertholletia excelsa), algumas leguminosas como Ingá (Inga edulis), Feijão de corda (Vigna unguiculata), entre outras espécies. Para a implantação e manutenção dessas culturas houve o preparo mecanizado, adubação e correção do solo.

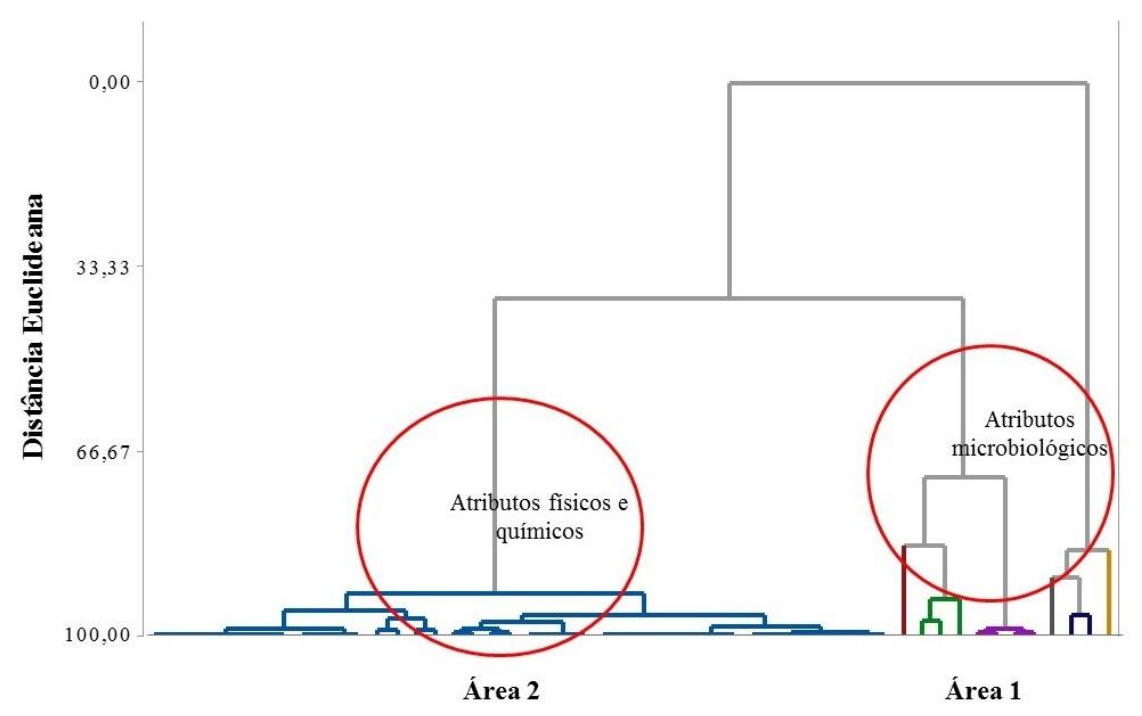


Figura 1. Dendrograma resultante da análise hierárquica de agrupamentos mostrando a formação de grupos, segundo os atributos analisados na comunidade de Macapazinho, PA. Área 1 = NFD, Área 2 = LAD.

Silva et al. (2017) e Mascarenhas et al. (2017) avaliando diferentes usos do solo também obtiveram resultados semelhantes, mostraram que quando os solos são bem manejados, os atributos do solo tendem a se manter em níveis adequados.

De acordo com Oliveira et al. (2017), o monitoramento de atributos de qualidade do solo pode servir como critério para detectar alterações impactantes na conservação do solo. Essas informações podem contribuir para o desenvolvimento de sistemas de manejos mais sustentáveis.

A variação dos atributos do solo na vegetação nativa é muito menor quando comparadas a dos solos de usos agrícolas e assim a vegetação nativa é um referencial para avaliação de solos incorporados a sistemas agrícolas. Nesta comparação pode-se observar as alterações de atributos do solo após a utilização agrícola e comparar os usos agrícolas, verificando-se qual deles apresenta maior sustentabilidade (FREITAS et al., 2015).

Nesta comparação é possível observar as alterações de atributos do solo após a utilização agrícola verificando-se, assim, maior sustentabilidade do solo utilizado como referência (NFD). É possível observar também que a área 2 (LAD) agregou alguns atributos do solo, o que significa que esse é um ambiente intermediário quanto à qualidade do solo em relação aos seus atributos físicos e químicos, demonstrando uma recuperação e conservação da qualidade desse solo.

\section{Considerações finais}

A implantação de culturas agrícolas em um Latossolo amarelo distrófico não altera os atributos químicos e físicos do solo, desde que o solo seja bem manejado, mantem-se os níveis adequado de fertilidade e a estrutura do solo. O uso das técnicas de multivariadas é eficiente para verificar indicadores de sustentabilidade, com base nos atributos químicos, físicos e microbiológico do solo, em cada ambiente estudado.

\section{Referências}

CAMPOS, M. C. C. et al. Topossequência de solos na transição Campos Naturais-Floresta na região de Humaitá, Amazonas. Acta Amazônica, v.42, n.3, p.387-398, 2012. 
métodos de análises de solo. 2.ed. Rio de Janeiro, Ministério da Agricultura e do abastecimento, 2011.

FREITAS, L. de et al. Técnicas multivariadas na avaliação de atributos de um Latossolo vermelho submetido a diferentes manejos. Revista Brasileira de Ciências Agrárias, v. 10, n. 1, 2015.

MASCARENHAS, A. R. P. et al. Atributos físicos e estoques de carbono do solo sob diferentes usos da terra em Rondônia, Amazônia Sul-Ocidental. Pesquisa florestal brasileira, v. 37, n. 89, p. 19-27, 2017.

MARQUES JÚNIOR, J. Caracterização de áreas de manejo específico no contexto das relações solo-relevo. Jaboticabal: Universidade Estadual Paulista Júlio de Mesquita Filho. 113p. Tese Livre-Docência. 2009.

MUGGLER, C. C. et al. Educação em solos: princípios, teoria e métodos. Revista Brasileira de Ciência do Solo, v. 30, n. 4, 2006.

Oliveira, I. A. Caracterização de solos sob diferentes ambientes na região Sul do Amazonas. Jaboticabal: Universidade Estadual Paulista Júlio de Mesquita Filho. 105p. Dissertação Mestrado, 2013.

OLIVEIRA, A. N. de et al. Atributos químicos de solo sob diferentes sistemas de uso e manejo no projeto de assentamento Veneza-São Domingos do Araguaia, PA. Revista Agroecossistemas, v. 9, n. 1, p. 170-179, 2017.

PEREIRA, S. S.; CURI, R. C. Meio ambiente, impacto ambiental e desenvolvimento sustentável: conceituações teóricas sobre o despertar da consciência ambiental. REUNIRRevista de Administração, Contabilidade e Sustentabilidade, v. 2, n. 4, p. 35-57, 2012.

REICHERT, J. M. et al. Variação temporal de propriedades físicas do solo e crescimento radicular de feijoeiro em quatro sistemas de ambiente. Pesquisa Agropecuária Brasileira, v.44, n.3, p.310-319. 2009.

SILVA, E. E. da et al. Determinação do Carbono da Biomassa Microbiana do Solo (BMS-C). EMBRAPA, Seropédica - RJ, Comunicado técnico 98, Julho, 2007a.

SILVA, E. E. da et al. Determinação do Nitrogênio da Biomassa Microbiana do Solo (BMSN). EMBRAPA, Seropédica - RJ, Comunicado técnico 96, Julho, 2007 b.

SILVA, S. A. et al. Variabilidade espacial de atributos químicos de um Latossolo VermelhoAmarelo húmico cultivado com café. Revista Brasileira de Ciência do Solo, v. 34, n.1, p.1522, 2010 a.

SILVA, M. O. et al. Discriminação de diferentes classes de solos irrigados com águas salinas, na região de Mossoró, RN, com o uso de análise multivariada. Ambiência, v. 6, n. 2, p. 261 $270,2010 \mathrm{~b}$. 
SILVA, Érika Andressa et al. Estrutura de solos em manejo conservacionista: Diagnóstico visual, laboratorial, caracterização e interrelações. Scientia agraria, v. 18, n. 3, p. 61-73, 2017. 